\title{
Nuevos desafíos para la industria alimentaria y los nuevos conocimientos acerca del tercer cerebro
}

\author{
New challenges for the food industry and new knowledge \\ about the third brain \\ Novos desafios para a indústria de alimentos e novos conhecimentos \\ sobre o terceiro cérebro
}

Mariela Maldonado ${ }^{1 *}$.

Recibido: 9 de julio de 2021. Aceptado para publicación: 18 de septiembre de 2021

Primero en línea: 23 de septiembre de 2021.

https://doi.org/10.35454/rncm.v4n4.326

\section{Resumen}

Los aditivos alimentarios son una de las sustancias de crecimiento exponencial en el mercado de la elaboración de alimentos debido a los hábitos de consumo excesivo de alimentos ultraprocesados y la necesidad de alimentos por la población creciente. Su uso y regulación está controlada sobre la base de supuestos toxicológicos tradicionales. Sin embargo, el nuevo paradigma de la influencia de la microbiota en la salud conduce a preguntarse cuáles son los desafíos de los aditivos alimentarios frente al rol de la microbiota en la salud. A la luz del rol que cumple la microbiota en la conformación del sistema inmune, la nutrición y los cambios que van desde el estado de salud hasta la aparición de la enfermedad que actualmente se conoce, los aditivos alimentarios deben ser revisados por nuevos estudios que involucran las diferentes ciencias "ómicas" con el fin de poder revaluar su inocuidad, sugerir dosis de uso frecuente y establecer recomendaciones nuevas para generar una legislación más actualizada. El objetivo de esta revisión es presentar los nuevos desafíos para la industria alimentaria y su relación con los nuevos conocimientos acerca del tercer cerebro.

Palabras clave: aditivos alimentarios, microbiota, sistema inmune, regulación, toxicología.

\section{Summary}

Food additives are among the substances experiencing exponential growth in the food processing market as a result of excess consumption of ultra-processed foods and the need for food by the growing population. Use and regulation are controlled based on traditional toxicological assumptions. However, under the new paradigm of how microbiota influences health, it is worth asking what is the relationship between food additives and the microbiota. Considering the role played by the microbiota in the conformation of the immune system, nutrition and disease-related changes currently known, food additives should be reviewed by new studies involving novel and different "omics" in order to assess their safety, suggest frequent use doses, and establish new recommendations for updated legislation. The aim of this review is to present the new challenges for the food industry and its relationship to new knowledge about the third brain.

Keywords: food additives, microbiota, immune system, regulation, toxicology.

\section{Resumo}

Os aditivos alimentares são uma das substâncias de uso alimentar em crescimento exponencial no mercado de processamento de alimentos devido ao consumo excessivo de alimentos ultraprocessados e à necessidade de alimentos por parte da população em crescimento. Seu uso e regulamentação são controlados com base em suposições toxicológicas tradicionais. Porém, o novo paradigma da influência da microbiota na saúde nos leva a questionar: quais são os desafios dos aditivos alimentares em relação ao papel da microbiota na saúde? Diante do papel desempenhado pela microbiota na conformação do sistema imunológico, na nutrição e nas mudanças que vão desde o estado de saúde ao surgimento de doenças que se conhece hoje, é que os aditivos alimentares devem ser revistos por novos estudos que envolvem as diferentes ciências ômicas, a fim de reavaliar sua segurança, sugerir doses mais utilizadas e estabelecer novas recomendações para gerar uma legislação mais atualizada. O objetivo desta revisão é apresentar os novos desafios para a indústria de alimentos e sua relação com os novos conhecimentos sobre o terceiro cérebro.

Palavras-chave: aditivos alimentares, microbiota, sistema imunológico, regulação, toxicologia.
Consejo Nacional de Investigaciones Científicas y Técnicas (CONICET), Argentina. Departamento de Ingeniería Química, Universidad Tecnológica Nacional-Facultad Regional de Mendoza (UTN-FRM). Mendoza, Argentina.
*Correspondencia: Mariela Maldonado marielabeatriz1972@yahoo.com.ar 


\section{INTRODUCCIÓN}

Los aditivos alimentarios son sustancias que se añaden a los alimentos para mantener o mejorar su inocuidad, su frescura, su sabor, su textura o su aspecto. El procesamiento de los alimentos sería imposible sin su existencia pues permite el logro de nuevos productos; es decir, la diversificación y el alargamiento de su vida de anaquel.

Por una parte, la tendencia del ser humano moderno a consumir alimentos cada vez más procesados y la necesidad de que esos alimentos abastezcan una población creciente, como el acceso a los alimentos de manera inocua, segura y deseable, ha producido un enorme impacto en el crecimiento de la industria de los aditivos alimentarios. Por otra parte, los nuevos conocimientos asociados con el enorme desarrollo tecnológico de las ciencias "ómicas" desde el proyecto del genoma humano y el desarrollo del proyecto del microbioma humano hacen surgir nuevos conocimientos sobre el rol de la microbiota en el campo de la salud y la alimentación. Se hace necesario probar todos los alimentos y sus aditivos para verificar su comportamiento frente a la microbiota, lo que pone a prueba los aspectos toxicológicos conocidos hasta el momento.

El objetivo de esta revisión es presentar los nuevos desafíos para la industria alimentaria y su relación con los nuevos conocimientos acerca del tercer cerebro.

\section{ANÁLISIS Y DISCUSIÓN}

Los cambios conductuales que se perciben en el consumidor cada vez más informado con respecto al valor nutricional, la inocuidad y la calidad de los alimentos exige un etiquetado nutricional más preciso, detallado y a la vez sencillo de interpretar. En paralelo, el desarrollo tecnológico y la necesidad de llegar a diferentes partes del mundo con poblaciones en crecimiento ha potenciado el consumo de los aditivos alimentarios debido al aumento de la demanda de productos alimentarios y a querer probar alimentos diferentes.

A futuro también se espera que los alimentos envasados aumenten la demanda, entre ellos, los pasabocas, las comidas congeladas, las comidas listas para comer, entre otros, dado el ritmo de vida vertiginoso que tienen los consumidores actuales cada día. Por su parte, la demanda creciente de los consumidores de productos nutritivos y de sabores exóticos y de alto valor también influirá en los fabricantes para que utilicen aditivos que mejoren la experiencia buscada por el consumidor ${ }^{(1)}$.

Adicionalmente, en el mercado de los alimentos hay un enorme interés por los productos orgánicos y natu- rales, y el consumidor tiende a elegir aditivos naturales $\mathrm{y}$ con propiedades funcionales que promuevan la salud. Esta creciente preocupación por la salud y el aumento de conciencia sobre los beneficios de varios productos alimenticios orgánicos hará que aumente la demanda de aditivos alimentarios que se obtienen de forma natural en el mercado mundial de aditivos alimentarios. Últimamente, se ha reforzado la adopción de productos de aditivos alimentarios naturales, incluidos los colorantes naturales, los conservantes orgánicos y los agentes aromatizantes, como el extracto de romero, edulcorantes a base de estevia, entre otros ${ }^{(1)}$. No obstante, el desarrollo exponencial y la ruta de aprobación de los mismos incluyen pruebas toxicológicas, las cuales en general son evaluadas por los organismos de control a nivel local, nacional e internacional.

A nivel internacional, el Comité Mixto FAO/WHO de Expertos en Aditivos Alimentarios (JECFA) evalúa cada aditivo alimentario con base en estudios científicos de todos los datos bioquímicos, toxicológicos y de otra índole, entre ellos los ensayos obligatorios en animales, los estudios teóricos y las observaciones en seres humanos. En cuanto a los ensayos toxicológicos, se deben realizar estudios de toxicidad aguda a corto y a largo plazo para determinar la absorción, distribución y excreción del producto y los posibles efectos perjudiciales tanto del propio aditivo como de sus subproductos para determinados niveles de exposición ${ }^{(2)}$.

Estos ensayos se basan en fundamentos de la medicina toxicológica de Paracelso (1493-1541). Él promocionó el término "toxicon" o "agente tóxico" como una entidad química y promulgó el siguiente enunciado que permanece como aforismo de la toxicología: "Todas las sustancias son venenosas; no hay ninguna que no sea tóxica. La correcta dosis diferencia al veneno del remedio". Además, este médico enunció los siguientes corolarios:

- La experimentación es esencial en el examen de la respuesta a cualquier tipo de sustancia.

- Se debe hacer una distinción entre el efecto y la propiedad tóxica y terapéutica de las sustancias.

- Las anteriores propiedades son en muchos de los casos indistinguibles y dependientes de la dosis.

- Se debe determinar el grado de especificidad de las sustancias en su efecto terapéutico o tóxico ${ }^{(3)}$.

El conocimiento toxicológico de los aditivos alimentarios está fuertemente basado en estos supuestos y, desde el punto de vista de la legislación y del control, se aplican a la realidad en la industria alimentaria sin 
mayores cuestionamientos. No obstante, de a poco van surgiendo enfoques más actualizados, como el estudio de la microbiota en la toxicología de los alimentos ${ }^{(4)}$. Esto último responde a que actualmente hay un nuevo paradigma de conocimientos que debería ser tenido en cuenta en la legislación y el control al momento de evaluar un aditivo alimentario como inocuo.

El actual conocimiento relaciona nuestra vitalidad con la microbiota o tercer cerebro ${ }^{(5)}$ del sistema digestivo, la cual es responsable de nuestra salud y de la regulación del sistema inmune ${ }^{(6,7)}$, e influye en el comportamiento ${ }^{(8)}$. Todo esto está profundamente ligado no sólo a nuestros hábitos alimentarios, al estilo de vida y a la actividad física ${ }^{(9)}$, sino también a la integridad y diversidad de la microbiota. Así mismo, esta se relaciona con la génesis de enfermedades neurodegenerativas diversas, además de la aparición de alergias alimentarias $^{(10,11)}$.

En condiciones normales de salud, los seres humanos albergan cantidades elevadas de diversos tipos de microorganismos que constituyen un importante ecosistema. La piel, la cavidad oral, el tracto vaginal y el tracto gastrointestinal son tejidos con las condiciones idóneas para permitir el crecimiento de estos microorganismos, pero es el tubo digestivo el que alberga al $95 \%$ del total de estas bacterias. La mayoría de ellas no es patógena (no origina enfermedades) y es imprescindible para que se realice una buena absorción de nutrientes, además de ejercer una importante barrera de defensa ${ }^{(6)}$ que confiere integridad a la mucosa intestinal y favorece la resistencia de la infección o proliferación de determinadas bacterias patógenas (parásitos y virus).

El compartimento gastrointestinal representa la superficie más grande del cuerpo humano, aproximadamente $200-400 \mathrm{~m}^{2}$, y está colonizada por alrededor de 100 billones de microorganismos, en su mayoría bacterias, colectivamente denominada "microbiota intestinal” ${ }^{(7)}$. Los 4 filos principales presentes en el tracto gastrointestinal humano son Firmicutes (49 \%-76 \%), Bacteroidetes (16\%-23\%) y, en porcentajes más bajos, Proteobacteria y Actinobacteria ${ }^{(12,13)}$. La diversidad y cantidad de bacterias varían a lo largo de la vida y a lo largo del tracto gastrointestinal: están influenciadas por diferencias en la genética del hospedador y por interacciones con el ambiente externo. Esto hace que la microbiota intestinal de cada individuo sea única ${ }^{(14,15)}$.

A través del tiempo de evolución se fundó una relación simbiótica entre el ser humano y la microbiota. Esta última facilita la digestión de sustancias y previene el establecimiento de patógenos, entre otras cosas, y a su vez recibe el beneficio de la disponibilidad de nutrientes y la temperatura corporal del hospedero $^{(14-17)}$. Por su parte, la información genética de las comunidades microbianas comensales y del hospedador humano interactúan constituyendo lo que se denomina "el metagenoma". Este proporciona información sobre el potencial genético de múltiples organismos individuales interactuando como una población, de tal forma que se puede considerar al metagenoma como una extensión del genoma humano ${ }^{(18)}$.

El papel desempeñado por la microbiota intestinal en el binomio del cerebro-intestino ha abierto nuevas vías para la investigación de los mecanismos moleculares subyacentes a enfermedades neurológicas y, en consecuencia, para el descubrimiento de una nueva generación de estrategias modificadoras de la enfermedad.

El desequilibrio entre la cantidad o proporción de microorganismos no patógenos y patógenos dentro del intestino humano se conoce como "disbiosis". Aunque inicialmente se asumió que la disbiosis solo interfería con la homeostasis intestinal, por lo que contribuía con las enfermedades intestinales crónicas, ahora se acepta que las modificaciones de la microbiota intestinal y, en consecuencia, la inflamación intestinal también pueden jugar un papel importante en el desarrollo de enfermedades neurológicas, incluida la enfermedad de Alzheimer, enfermedad de Parkinson, trastorno del espectro autista (ASD) y esquizofrenia ${ }^{(19,20)}$. Para Moss y colaboradores en $2016^{(20)}$, la microbiota actuaría en la dirección de alimentos, activación de ciertos fármacos y producción de ácidos grasos de cadena corta (SCFA), que ayudan a modular la expresión génica con la inhibición de la desacetilación de las proteínas histonas, generación de sustancias antiinflamatorias y actuación fundamental en la inducción, entrenamiento y función del sistema inmune.

La comunicación entre la microbiota intestinal y el cerebro es bidireccional. Esta se puede establecer a través de varios mecanismos. Uno de estos implica la inhibición de la actividad de la histona desacetilasa, enzima inducida por los SCFA como butirato, propionato y acetato, que son los productos finales de la fermentación de polisacáridos por microorganismos intestinales. En las enfermedades de Alzheimer y Parkinson se ha observado que la concentración de estos ácidos es menor y también existen desregulaciones transcripcionales por la baja acetilación de las histonas, entre otras características $^{(20-22)}$. Además, los desequilibrios de la microbiota intestinal pueden conducir a una serie de patologías como obesidad ${ }^{(23)}$, diabetes tipos 1 y 2 , enfer- 
medad inflamatoria intestinal (EII), cáncer colorrectal (CCR) e inflamación/inmunosenescencia en los ancianos, entre otras ${ }^{(24,25)}$. Por estas razones, la Sociedad Española de Prebióticos y Probióticos ha explicado que cada vez se da mayor importancia a la modulación de la microbiota intestinal mediante el empleo de probióticos, prebióticos y simbióticos para tratar diversas enfermedades, principalmente problemas gastrointestinales ${ }^{(26)}$, así como también está surgiendo interés por el estudio de sustancias psicobióticas ${ }^{(27,28)}$; es decir, sustancias capaces de regular los estados de ánimo mediante la modificación del control génico de probióticos que conviven en el tracto intestinal.

Específicamente para aditivos alimentarios, se ha encontrado que los emulsionantes dietéticos como la carboximetilcelulosa (CMC) y polisorbato 80 (P80) alteran la composición de la microbiota intestinal e inducen una inflamación crónica de bajo grado, lo que en última instancia conduce a desregulaciones en ratones y cambios en su conducta ${ }^{(29)}$. En el caso de los edulcorantes, varios se han sometido a estudios exhaustivos al respecto y se ha encontrado que la sacarina en humanos y ratones, la sucralosa en ratones y la estevia en humanos cambian levemente la composición de la microbiota ${ }^{(30)}$.

Estos ejemplos de cómo los aditivos alimentarios pueden modificar la microbiota son una alerta en la abundancia de aditivos alimentarios que se consumen en la actualidad. Si la modificación de la microbiota puede producir una disbiosis y, con ello, un cambio perjudicial de la salud, cabe preguntarse qué pasa con los aditivos que son incorporados cada vez más en los alimentos industrializados y cuyo consumo aumenta progresivamente en la población humana.

Hasta ahora, desde el punto de vista del mercado de los aditivos, los factores que se espera que restrinjan el crecimiento del mercado son la aplicación de políticas regulatorias estrictas, así como otros problemas relacionados con la salud como alergias u otros que pueden surgir debido a la utilización de aditivos. No obstante, se prevé que la creciente cantidad de avances innovadores relacionados con los aditivos alimentarios y los esfuerzos continuos de investigación y desarrollo abrirán oportunidades de crecimiento para los actores clave que operan en el mercado global durante los próximos 10 años ${ }^{(1)}$. Aprovechar el impulso de la investigación y desarrollo de aditivos debería contemplar este nuevo paradigma que posiciona a la microbiota como el centro de la cuestión en la promoción de la salud humana.
Por esto, todos estos nuevos conocimientos conducen a plantear cómo se comportan los aditivos alimentarios frente a la microbiota, solos o combinados, tal como se consumen en los alimentos.

\section{CONCLUSIÓN}

Al ser tan específica y tan importante la influencia de la microbiota en el estado de salud del hospedero, los ensayos de toxicidad aguda y crónica realizados en animales quedan limitados, en primer lugar, porque la microbiota de los animales de laboratorio no es necesariamente la misma que la del ser humano, como tampoco lo es su metabolismo; y en segundo lugar, porque cada individuo presenta una microbiota diferente y el estado de no toxicidad y de salud podría verse modificado por la misma. Por estas razones, antes de los ensayos en humanos deberían implementarse estudios a nivel microbiológico in vitro e in vivo, combinados con estudios de proteómica, metabolómica, interactoma y reactoma de cada ecosistema microbiano frente a un hospedador a fin de dilucidar si un aditivo es benéfico o no para el huésped.

Este nuevo desafío deviene del avance tecnológico en lo referente a las ciencias "ómicas" relacionadas con el nuevo paradigma clínico de tratamiento de enfermedades tratadas mediante la utilización de agentes bioterapéuticos: prebióticos, probióticos y simbióticos ${ }^{(18)}$. Entonces, debería estudiarse si los aditivos alimentarios actúan como prebióticos y en último caso son inocuos, aunque no sean favorecedores de la promoción de los probióticos. Es decir que este tipo de estudios deberían ser contemplados previamente a la aprobación de una sustancia como aditivo alimentario, como también deberían enviarse a revisión todos los aditivos aprobados con anterioridad a la luz de estos nuevos conocimientos.

Entonces, la premisa de la Organización Mundial de la Salud (OMS) de que "la utilización de aditivos alimentarios solamente está justificada si responde a una necesidad tecnológica, no induce a error al consumidor y se emplea con una función tecnológica bien definida, como la de conservar la calidad nutricional de los alimentos o mejorar su estabilidad"(2) no sería suficiente para justificar la producción y uso de un aditivo. Si el consumo de un aditivo alimentario causara una alteración negativa de la microbiota y sus interacciones, debería ser estudiado con mayor profundidad teniendo en cuenta los nuevos conocimientos. En otras palabras, ante la duda, los estudios de cada uno de los aditivos 
alimentarios frente a la microbiota deberían ser ampliados y posiblemente obligatorios antes de que un aditivo sea aprobado por la legislación.

\section{Agradecimientos}

Al CONICET y la UTN-FRM de Argentina.

\section{Declaración de autoría}

Mariela Maldonado ideó y es responsable de la investigación, redacción del manuscrito, revisión y envío.

\section{Fuente de financiación}

Este manuscrito fue financiado por los Secretarios de Ciencia y Tecnología (SECyT, UTN-FRM) PID 6571 "Elaboración de cerezas en conserva reducidas en valor calórico y coloreadas con colorantes naturales".

\section{Conflicto de intereses}

La autora declara no tener ningún conflicto de intereses.

\section{Referencias bibliográficas}

1. Global food additives market, drivers, restraints, opportunities, threats, trends, applications, and growth forecast to 2029 [internet]. MarketResearch.Biz; 2021 [consultado el 1 de junio de 2021]. Disponible en: https://marketresearch.biz/ report/food-additives-market/

2. Aditivos alimentarios. World Health Organization; 2018 [consultado el 1 de junio de 2021]. Disponible en: https://www. who.int/es/news-room/fact-sheets/detail/food-additives

3. Valle Vega P, Florentino BL. Toxicología de alimentos [internet]. México, D.F.: Instituto Nacional de Salud Pública, Centro Nacional de Salud Ambiental; 2000. [consultado el 1 de junio de 2021]. Disponible en: https://www.fio.unicen.edu.ar/ usuario/gmanrique/images/Toxicologia_de_Alimentos VegaFlorentino.pdf

4. Mellado Durán E, A, MI, AM. Importancia de la microbiota del tracto gastrointestinal en toxicología alimentaria. En: Cameán AM, Repetto M (editores). Toxicología alimentaria. Madrid: Díaz de Santos, 2012. p. 1-24.

5. Greenlaw P, Ruggiero M, Greenlaw D. Your third brain: The revolutionary new discovery to achieve optimum health. Extraordinary Wellness Publishing; 2015.

6. Thursby E, Juge N. Introduction to the human gut microbiota. Biochem J. 2017;474(11):1823-1836. doi: 10.1042/ BCJ20160510

7. Fung TC, Olson CA, Hsiao EY. Interactions between the microbiota, immune and nervous systems in health and disease. Nat Neurosci. 2017;20(2):145-155. doi: 10.1038/ nn. 4476
8. Ruiz-Briseño MR, Sánchez-Reyes K, Alvarez-Zavala M, González-Hernández LA, Ramos-Solano Metal.Homeostasis intestinal: colaboracion del sistema inmune con la microbiota. Rev Med MD. 2018;4:337-340.

9. Oriach CS, Robertson RC, Stanton C, Cryan JF, Dinan TG. Food for thought: The role of nutrition in the microbiota-gutbrain axis. Clinical Nutrition Experimental. 2016;6:25-38. Doi: 10.1016/j.yclnex.2016.01.003

10. Castellanos N, Diez GG, Antúnez-Almagro C, Bailén M, Bressa C, González Soltero R, et al. A Critical Mutualism - Competition Interplay Underlies the Loss of Microbial Diversity in Sedentary Lifestyle. Front Microbiol. 2020;10:3142. doi: 10.3389/fmicb.2019.03142

11. Navia-López LA, Ignorosa-Arellano KR, Zárate-Mondragón FE, Cervantes Bustamante R, Toro-Monjaraz EM, Cadena León JF, et al. Microbiota gastrointestinal y su relación con la alergia. Acta Pediatr Mex. 2020;41(3):135-47.

12. Matsuoka K, Kanai T. The gut microbiota and inflammatory bowel disease. Semin Immunopathol. 2015;37(1):47-55. doi: 10.1007/s00281-014-0454-4

13. Sartor RB, Mazmanian SK. The Intestinal Microbiota in Inflammatory Bowel Diseases. Am J Gastroenterol. 2012;1(Suppl):15-21. doi: 10.1038/ajgsup.2012.4

14. Brown EM, Sadarangani M, Finlay BB. The role of the immune system in governing host-microbe interactions in the intestine. Nat Immunol. 2013;14(7):660-7. doi: 10.1038/ni.2611

15. Hooper LV, Macpherson AJ. Immune adaptations that maintain homeostasis with the intestinal microbiota. Nat Rev Immunol. 2010;10(3):159-69. doi: 10.1038/nri271

16. Goto Y, Ivanov II. Intestinal epithelial cells as mediators of the commensal-host immune crosstalk. Immunol Cell Biol. 2013;91(3):204-14. doi: 10.1038/icb.2012.80

17. Maynard CL, Elson CO, Hatton RD, Weaver CT. Reciprocal interactions of the intestinal microbiota and immune system. Nature. 2012;489(7415):231-41. doi: 10.1038/nature11551

18. Hernández Hernández A, Coronel Rodríguez C, Monge Zamorano M, Quintana Herrera C. Microbiota, probióticos, prebióticos y simbióticos. Pediatría Integral. 2015;19(5):337-54.

19. Houser MC, Tansey MG. The gut-brain axis: is intestinal inflammation a silent driver of Parkinson's disease pathogenesis? NPJ Parkinsons Dis. 2017;3:3. doi: 10.1038/s41531-0160002-0

20. Moos WH, Faller DV, Harpp DN, Kanara I, Pernokas J, Powers WR, et al. Microbiota and Neurological Disorders: A Gut Feeling. Biores Open Access. 2016;5(1):137-45. doi: 10.1089/biores.2016.0010

21. Chuang DM, Leng Y, Marinova Z, Kim HJ, Chiu CT. Multiple roles of HDAC inhibition in neurodegenerative conditions. Trends Neurosci. 2009;32(11):591-601. doi: 10.1016/j. tins.2009.06.002

22. Dinan TG, Cryan JF. The Microbiome-Gut-Brain Axis in Health and Disease. Gastroenterol Clin North Am. 2017;46(1):77-89. doi: 10.1016/j.gtc.2016.09.007 
23. Basain Valdés JM, Valdés Alonso MC, Miyar Pieiga E, Linares Valdés $\mathrm{H}$, Martínez Izquierdo A. Alteraciones en la microbiota intestinal por la dieta y su repercusión en la génesis de la obesidad. Medisan. 2015;19(12):1536-46.

24. Tibbs TN, Lopez LR, Arthur JC. The influence of the microbiota on immune development, chronic inflammation, and cancer in the context of aging. Microb Cell. 2019;6(8):324334. doi: $10.15698 / \mathrm{mic} 2019.08 .685$

25. Serra D, Almeida LM, Dinis TCP. Dietary polyphenols: A novel strategy to modulate microbiota-gut-brain axis. Trends Food Sci Technol. 2018;78:224-33. Doi: 10.1016/j. tifs.2018.06.007

26. Peterson CT, Sharma V, Elmén L, Peterson SN. Immune homeostasis, dysbiosis and therapeutic modulation of the gut microbiota. Clin Exp Immunol. 2015;179(3):363-77. doi: $10.1111 /$ cei. 12474
27. de Carvalho Furtado C, Lima Bispo da Silva A, Matias Walfall A. Psicobióticos: uma ferramenta para o tratamento no transtorno da ansiedade e depressão? UNILUS Ensino e Pesquisa. 2018;15:137-51.

28. Schmidt C. Mental health: thinking from the gut. Nature. 2015;518(7540):S12-5. doi: 10.1038/518S13a

29. Holder MK, Peters NV, Whylings J, Fields CT, Gewirtz AT, Chassaing B, et al. Dietary emulsifiers consumption alters anxiety-like and social-related behaviors in mice in a sexdependent manner. Sci Rep. 2019;9(1):172. doi: 10.1038/ s41598-018-36890-3

30. Ruiz-Ojeda FJ, Plaza-Díaz J, Sáez-Lara MJ, Gil A. Effects of Sweeteners on the Gut Microbiota: A Review of Experimental Studies and Clinical Trials. Adv Nutr. 2019;10(suppl_1):S31S48. doi: 10.1093/advances/nmy037 